UDC 621.317

LBC 31.221-5

\title{
ELECTRONIC MODULE FOR PHOTOPLETHYSMOGRAPHY AND PULSE OXIMETRY
}

\author{
Igor B. Isupov \\ Volgograd State University, Volgograd, Russian Federation \\ Rimma Sh. Zatrudina \\ Volgograd State University, Volgograd, Russian Federation
}

\begin{abstract}
Methods of pulse oximetry and photoplethysmography are widely used in clinical medicine and practice of biomedical research outside the clinic. The advantages of the method are high efficiency of preparation for the diagnostic procedure, non-invasive, complete safety for health. The method provides the possibility of unlimited long - term monitoring of the dynamics of saturation and desaturation of hemoglobin. In combination with the above, subject to the qualitative registration of photoplethysmograms, the method allows the calculation and analysis of a number of indicators of peripheral blood circulation: the total pulse blood flow to the study region, the tone of arterial vessels of different diameters, the state of capillary blood flow. Most modern foreign models of pulse oximeters are integrated devices that provide measurement of a very limited set of physiological parameters: the percentage of hemoglobin oxygen saturation and pulse rate. The range of pulse oximeters containing the interface with the personal computer and providing operational calculation of indicators of peripheral photoplethysmograms is small. Such devices have a fairlyhigh cost and are not portable.

The development of domestic integrated electronic devices is extremely relevant, allowing along with obtaining information about blood oxygenation to perform continuous monitoring of peripheral blood circulation. The authors developed an electronic module that provides long-term registration of photoplethysmograms in the infrared and red areas of the light spectrum. The electronic module is made exclusively with the use of domestic active radio elements discrete semiconductor devices and integrated circuits. The device was developed in full compliance with the import substitution policy pursued by the President and the Government of the Russian Federation.

The advantage of the device is the "open architecture" of the electronic circuit, which provides four analog signals suitable for further digitization. The signals contain information about pulsating and non-pulsating components of the optical density of biological tissue in the infrared and red ranges of the spectrum. The device has an electronic filter of mains interference.

Tests of the device demonstrated high quality of photoplethysmograms. This makes it possible tosuccessfullyuse the device for studies of hemoglobin oxygenation and peripheral hemodynamics.

Key words: photoplethysmography, pulse oximetry, operational amplifier, trigger, a multiplexer, a light emitting diode, a photodiode, an electrical filter.
\end{abstract}

\section{ЭЛЕКТРОННЫЙ МОДУЛЬ ДЛЯ ФОТОПЛЕТИЗМОГРАФИИ} И ПУЛЬСОКСИМЕТРИИ

\author{
Игорь Борисович Исупов \\ Волгоградский государственный университет, г. Волгоград, Российская Федерация \\ Римма Шикрулловна Затрудина \\ Волгоградский государственный университет, г. Волгоград, Российская Федерация
}


Аннотация. Методики пульсоксиметрии и фотоплетизмографии получили широкое распространение в клинической медицине и практике медико-биологических исследований вне клиники. Достоинствами метода являются высокая оперативность подготовки обследуемого к диагностической процедуре, неинвазивность, полная безопасность для здоровья. Метод обеспечивает возможность неограниченно длительного мониторинга динамики сатурации и десатурации гемоглобина. В сочетании с перечисленным, при условии качественной регистрации фотоплетизмограмм метод позволяет осуществлять расчет и анализ ряда показателей периферического кровообращения: суммарного пульсового притока крови в исследуемый регион, тонуса артериальных сосудов различного диаметра, состояния капиллярного кровотока. Большинство современных зарубежных моделей пульсоксиметров представляют собой интегральные устройства, обеспечивающие измерение очень ограниченного набора физиологических параметров: процент насыщения гемоглобина кислородом и частоту пульса. Ассортимент пульсоксиметров, содержащих интерфейс с персональным компьютером и обеспечивающих оперативный расчет показателей периферических фотоплетизмограмм, невелик. Подобные приборы имеют довольно высокую стоимость и не являются портативными.

Исключительно актуальна разработка отечественных интегральных электронных устройств, позволяющих наряду с получением информации об оксигенации крови выполнять непрерывный мониторинг периферического кровообращения.

Авторами разработан электронный модуль, обеспечивающий длительную регистрацию фотоплетизмограмм в инфракрасном и красном участках светового спектра. Электронный модуль выполнен исключительно с применением отечественных активных радиоэлементов - дискретных полупроводниковых приборов и интегральных микросхем. Разработка устройства проведена в полном соответствии с политикой импортозамещения, проводимой Президентом и Правительством Российской Федерации.

Достоинством устройства является «открытая архитектура» электронной схемы, которая обеспечивает получение четырех аналоговых сигналов, пригодных для дальнейшей оцифровки. Сигналы содержат информацию о пульсирующем и непульсирующем компонентах оптической плотности биологической ткани в инфракрасном и красном диапазонах спектра. Прибор имеет электронные фильтры сетевых помех.

Испытания устройства продемонстрировали высокое качество фотоплетизмограмм. Это позволяет успешно использовать прибор для исследований оксигенации гемоглобина и периферической гемодинамики.

Ключевые слова: фотоплетизмография, пульсоксиметрия, операционный усилитель, триггер, мультиплексор, светоизлучающий диод, фотодиод, электрический фильтр.

В последние десятилетия прошлого века для функциональной диагностики состояния сердечно-сосудистой, дыхательной систем и, в известной мере, функциональных особенностей системы крови широкое распространение получили пульсоксиметры - приборы, предназначенные для определения относительного насыщения гемоглобина крови кислородом в естественных условиях жизнедеятельности человека и в динамике выполнения им различных функциональных проб [4, 5, 6, 10].

По сравнению с ранее использовавшимися для решения указанной задачи фотооксигемографами, получившими распространение в середине - последней четверти прошлого века, современные, интегральные пульсоксиметры позволяют получать высококачественные кривые периферического артериального пульса, обусловленного периодической деятельностью сердца - фотоплетизмограммы (ФПГ) [7, 9]. Контур, форма ФПГ в значительной степени напоминает контур кривой периферического пульса (сфигмограммы), получа- емой с использованием механических датчиков давления, преобразующих колебания стенки пульсирующей артерии в электрический сигнал $[3,5,8]$. Благодаря последнему обстоятельству, наряду с со своим традиционным применением, пульсоксиметрическая приставка может быть использована в качестве источника первичной биологической информации о частоте сердечных сокращений и естественной вариабельности ритма сердца человека [2]. Иначе говоря, современный пульсоксиметр может выполнять ценную побочную функцию: использоваться для сопутствующей фотоплетизмографии, позволяя получать ценную диагностическую информацию о пульсовом кровенаполнении периферических регионов, тонусе их артерий и артериол, а также может найти применение для реализации методики вариационной пульсометрии, успешно конкурируя с электрокардиографическими устройствами, традиционно используемыми для получения информации об изменчивости ритма сердца [2]. 
Однако стационарные высококачественные пульсоксиметры, имеющие интерфейс с микроЭВМ и реализующие вышеперечисленные функции довольно дороги и не всегда доступны. В соответствие с политикой импортозамещения в различных отраслях науки и техники актуальна разработка отечественных приборов, пригодных для выполнения функционально-диагностических процедур в клинических и медико-биологических лабораториях.

Цель работы: создание универсальной пульсоксиметрической-фотоплетизмографической приставки, позволяющей получать информацию о сатурации артериального гемоглобина кислородом, функциональном состоянии периферической гемодинамики и вегетативных регуляциях хронотропной функции сердца.

Материалы и методы. В работе использована методика последовательного линейного проектирования электронных схем, содержащих цифровые и аналоговые интегральные микросхемы (ИМС).

Конструирование электронного модуля выполнено в четыре этапа.

Первый, расчетно-проектировочный, этап осуществлялся согласно стандартным требованиям к расчетам аналоговых устройств, содержащих операционные усилители (ОУ). Конструирование цифровых узлов прибора выполнено авторами самостоятельно.

Второй этап представлял собой макетирование всех узлов устройства, выполненное поблочно, без применения пайки. На указанном этапе проведена коррекция номиналов пассивных радиоэлементов, обеспечивающих необходимые коэффициенты усиления каналов прибора, частотный диапазон его аналогового тракта, а также фильтрацию сетевых помех с частотой 50 Гц. На данном этапе разработана конструкция клипсообразного датчика, обеспечивающего получение информации об оптической плотности тканей дистальной фаланги указательного пальца человека.

На третьем этапе выполнено проектирование печатной платы устройства, монтаж и окончательная отладка электронного модуля с помощью контрольно-измерительных приборов.

На четвертом этапе осуществлена стыковка выходов электронного модуля с анало- говыми входами универсального микроконтроллера «АРДУИНО-НАНО», создание драйвера устройства, испытания прибора в реальных условиях.

Для создания электронного модуля использованы приборы: электронно-лучевой осциллоскоп (осциллограф) «С1-65A», универсальный цифровой мультиметр (авометр) «MastechMY65».

Результаты и их обсуждение (описание устройства). Блок-схема электронного модуля представлена на рисунке 1. Принцип действия устройства является общепринятым для современных пульсоксиметров, работающих в проходящем (неотраженном) свете, и основан на получении электрического сигнала, являющегося эквивалентом интенсивности светового потока, проходящего через биологический объект, оптическая плотность которого периодически изменяется вследствие пульсовых колебаний кровенаполнения артерий данного объекта $[4,5]$.

Биологическим объектом является дистальная фаланга указательного пальца обследуемого, на которую одевается клипсообразный датчик информации - оптрон с открытым оптическим каналом, содержащий два источника оптического излучения (светодиоды диоды с различными длинами волн излучения) и два идентичных, предварительно подобранных по параметрам фотоприемника - кремниевые фотодиоды ФД-256.

Для получения информации о насыщении гемоглобина кислородом использован следующий технический прием: попеременная импульсная подача светового потока двумя излучателями на двух различных длинах волн: 635 нм (световой поток в красной области видимого спектра, создаваемый светодиодом КИПД-2000) и 920 нм (световой поток в инфракрасной области, создаваемый светодиодом АЛ115А).

В качестве генератора электрических импульсов, обеспечивающих попеременное включение обоих светодиодов использован несимметричный мультивибратор (цифровая ИМС К561ЛА7 или К561ЛЕ5). Прямоугольные импульсы с выхода ИМС поступают на С-вход D-триггера (цифровая ИМС К561TM2), включенного в качестве счетчика-делителя частоты на 2. В результате на прямом и инверсном 
выходах триггера появляются импульсы вдвое меньшей частоты, равной длительности и противоположные по фазе.

Таким образом, с помощью несложных схемотехнических приемов обеспечена абсолютно равная длительность «красного» и «инфракрасного» импульсов оптического излучения.

К прямому и инверсному выходам триггера подключены транзисторные ключи (транзисторы КТ315Г; на рис. 1 не показаны), являющиеся усилителями тока и обеспечивающие необходимую интенсивность светового потока в обоих диапазонах. В коллекторные цепи соответствующих транзисторов включены красный и инфракрасный светоизлучающие диоды.

Прошедший через биологический объект и воспринятый фотоприемниками (фотодиоды ФД-256) световой поток преобразуется в сложный электрический сигнал, содержащий постоянную и переменную составляющую. Первая отражает оптическую плотность непульсирующих тканей биологического объекта, вторая - оптическую плотность пульсиру- ющих тканей (артерио-артериолярную составляющую).

Импульсный электрический сигнал сложной формы с выхода фотоприемников подвергается предварительному усилению (аналоговая ИМС - широкополосный ОУ КР140УД708). Коэффициент усиления ОУ составляет 2-3.

После предварительного усиления сигнал подвергается демультиплексированию (электронной селекции), таким образом, что дальнейшее усиление его компонента, являющегося эквивалентом светового потока, полученного под действием импульса красного источника света, обеспечивается одним основным усилительным каналом приставки, а усиление компонента, полученного под действием импульса инфракрасного излучателя, обеспечивается синхронно и независимо от первого вторым основным усилительным каналом прибора. В качестве селектора сигналов использован сдвоенный аналоговый мультиплексор К561КП1.

Оба основных усилительных канала (красный и инфракрасный) имеют идентичную

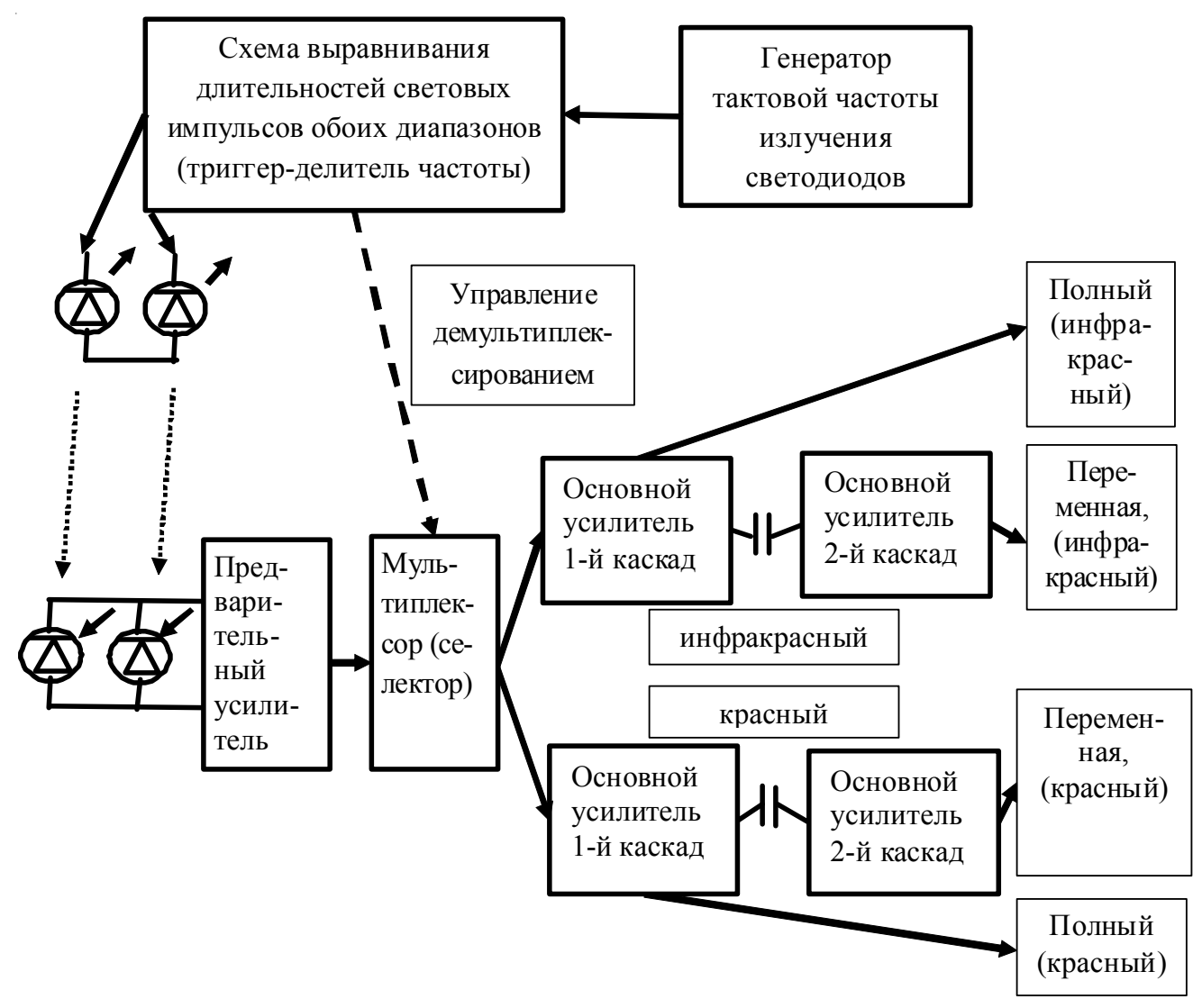

Рис. 1. Блок-схема электронного модуля 
И.Б. Исупов, Р.Ш. Затрудина. Электронный модуль для фотоплетизмографии и пульсоксиметрии

электрическую схему и представляют собой двухкаскадные широкополосные усилители напряжения, выполненные на интегральных ОУ (КР544УД1А), включенных по схеме инвертирующего усиления.

С выходов первых каскадов обоих основных усилителей полный электрический сигнал поступает непосредственно на аналоговые входы микроконтроллера АРДУИНО-НАНО и, через разделительный конденсатор, на входы вторых каскадов основных усилителей. Емкостная связь между первым и вторым каскадами каждого основного усилителя необходима для выделения переменной (пульсовой) составляющей полного сигнала фотоплетизмограмм.

Полный коэффициент усиления обоих каскадов основных усилительных трактов в каждом диапазоне равен 200-300.

Каждый тракт основного усиления сигнала содержит двухзвенный пассивный электрический RC-фильтр нижних частот, предназначенный для демодуляции импульсного сигнала, получаемого на выходе мультиплексора-селектора, а также для снижения уровня сетевой наводки («фона переменного тока» с частотой сети 50Гц) до приемлемого уровня.
После усиления вторым каскадом переменная составляющая фотоплетизмографических сигналов красного и инфракрасного диапазонов поступает на соответствующие аналоговые входы программируемого микроконтроллера АРДУИНО-НАНО.

Испытания разработанного электронного модуля выявили высокое качество фотоплетизмограмм, получаемых с указательной фаланги пальца обследуемых, в течение длительного времени (рис. 2).

Заключение. Применение отечественных цифровых интегральных микросхем серий К561, малошумящих операционных усилителей серий К140 (КР140) и К544 (КР544) позволяет создавать малогабаритные электронные модули, обеспечивающие высококачественную регистрацию фотоплетизмограмм в красном и инфракрасном диапазонах светового спектра. Это позволяет при наличии соответствующего математического алгоритма отслеживать процесс сатурации/десатурации гемоглобина крови в различных условиях жизнедеятельности человека.

Разработанный авторами электронный модуль, содержащий всего восемь цифро-

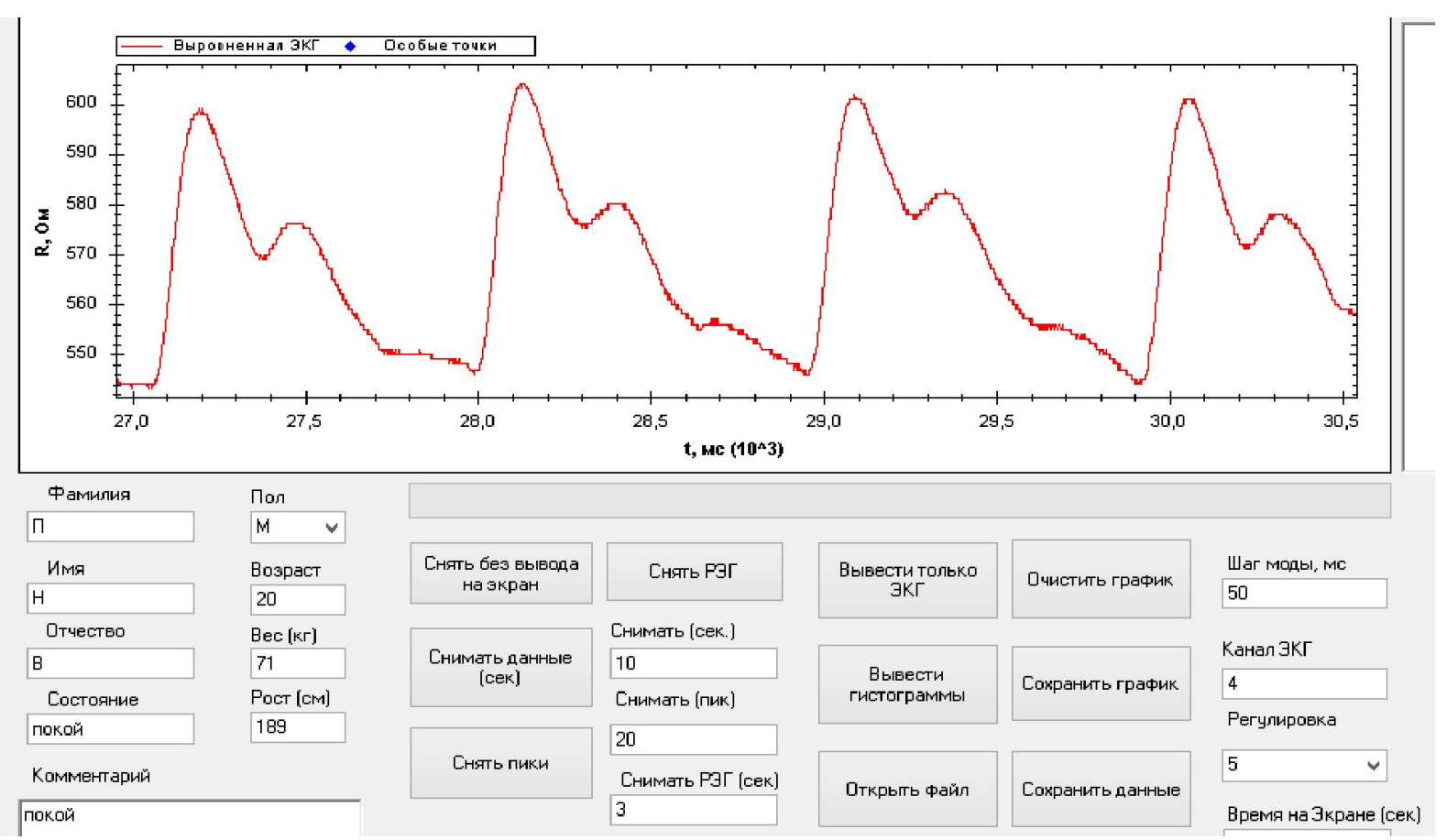

Рис. 2. Фрагмент тестовой записи ФПГ указательного пальца правой руки обследуемого П. в состоянии физиологического покоя. (На фрагменте показаны ФПГ на 27-31-й секундах записи,

полной длительностью 1 мин. Для регистрации использован программный продукт, описанный в [8]) 
вых и аналоговых ИМС, обеспечивает стабильную регистрацию фотоплетизмограмм в течение длительного времени у обследуемых, имеющих резко выраженные индивидуальные особенности оптических пульсовых кривых. Это открывает возможности применения предлагаемого устройства для мониторинговых исследований колебаний регионарного пульсового кровенаполнения артерий, тонуса резистивных сосудов конечностей, в частности у лиц с нейроциркуляторными расстройствами гемодинамики и, вероятно, болезнью Рейно. Важнейшим следствием мониторинговой регистрации ФПГ может являться вариационно-статистический и гармонический анализ вариабельности регионарного сосудистого тонуca, успешно применяемый в последние годы для анализа реограмм, углубленное изучение спектров мощности дыхательных волн и волн Майера, что актуально для ранней диагностики различной патологии периферического кровообращения $[1,3]$.

Электронный модуль может найти применение для исследований вариабельности сердечного ритма во внебольничных условиях, являясь недорогой альтернативой компьютеризованным электрокардиографическим мониторам.

\section{СПИСОК ЛИТЕРАТУРЫ}

1. Гармонические перспективы реографии / С. В. Ревенко // Нервно-мышечные болезни. 2012. - № 4. - C. 8-18.

2. Исследование погрешностей данных фотоплетизмограммы для анализа вариабельности сердечного ритма / М. И. Стригина [и др.] // Биомедицина. - 2013. - № 4. - С. 139-148.

3. Кардиодиагностика на основе анализа фотоплетизмограмм с помощью двухканального плетизмографа / М. Галкин [и др.] // Фотоника. - 2008. № 3. - C. 30-35.

4. Мониторинг дыхания: пульсоксиметрия, капнография, оксиметрия / И.А. Шурыгин // СПб.: «Невский Диалект»; М.: «Издательство БИНОМ». 2000. $-301 \mathrm{c}$.

5. Физические основы оптической оксиметрии / Д. А. Рогаткин // Медицинская физика. 2012. - № 2. - С. 97-114.

6. Acceleration plethysmography to evaluate aging effect in cardiovascular system / H.Takada [et al.] // Med. ProgressTechnol. - 1997. - V. 21.p. 205.

7. Assessment of vasoactive agents and vascular aging by second derivative of the photoplethysmograph waveform / K.Takazawa [et al.] // Hypertension. - 1998.v. 32. - p. 365 .

8. Automated analysis of plethysmograms for functional studies of hemodynamics / R.Sh. Zatrudina [et al.]// Proc. SPIE 10717, Saratov Fall Meeting 2017: Laser Physics and Photonics XVIII; and Computational Biophysics and Analysis of Biomedical Data IV, 1071727 (26 April 2018); doi: 10.1117/12.2317482; https://doi.org/ $10.1117 / 12.2317482$.

9. Correlation between wave components of the second derivative of plethysmogram and arterial distensibility / I.Imanaga [et al.] // Jpn. Heart J. - 1998. v. 39. - p. 775.

10. Noninvasive assessment of the digital volume pulse. Comparison with the peripheralpressure pulse. / S.C. Millasseau [et al.] // Hypertension. - 2000. v. $36 .-$ p. 952 .

\section{REFERENCES}

1. Revenko S. V. (2012)Rheography: Harmonic perspectives.Neuromuscular diseases. - Vol. 4. - P. 8 18 [Rus., Eng. Abstr.].

2. Strigina M. I., Chaivanov D.B., Chudina J. A. (2013) Study of errors of photoplethysmogram data for the analysis of heart rate variability.Biomedicine.Vol. 4. - P. 139-148 [Rus., Eng. Abstr.].

3. Galkin M., ZmievskoyG.,Laryushin A., Novikov V. (2008) Cardiodiagnostics based on the analysis of photoplethysmograms using a two-channel plethysmograph. Photonics. - Vol. 3. - P. 30-35. [Rus., Eng. Abstr.].

4. ShuryginI.A. (2000)Breath monitoring: pulse oximetry, capnography, oximetry / SPb: «The Neva Dialect»; Moscow: «IZDATEL'stvo BINOM».-301 p. [Rus., Eng. Abstr.].

5. Rogatkin D. (2012) Physical basis of optical oximetry.Medical physics. -Vol. 2. - P. 97-114 [Rus., Eng. Abstr.].

6. Takada H. et al. (1997) Acceleration plethysmography to evaluate aging effect in cardiovascular system. Med. Progress Technol., v. 21, p. 205.

7. Takazawa K. et al. (1998) Assessment of vasoactive agents and vascular aging by second derivative of the photoplethysmograph waveform. Hypertension, v. 32, p. 365.

8. ZatrudinaR.Sh.,IsupovI.B., GribkovV.Yu. (2018) Automated analysis of plethysmograms for functional studies of hemodynamics. Proc. SPIE 10717, Saratov Fall Meeting 2017: Laser Physics and 
И.Б. Исупов, Р.Ш. Затрудина. Электронный модуль для фотоплетизмографии и пульсоксиметрии

Photonics XVIII; and Computational Biophysics and Analysis of Biomedical Data IV, 1071727 (26 April 2018); doi: 10.1117/12.2317482; https://doi.org/10.1117/ 12.2317482 .

9. Imanaga I. et al. (1998) Correlation between wave components of the second derivative of plethysmogram and arterial distensibility. Jpn. Heart J., v. 39, p. 775 .

10. Millasseau S.C. et al. (2000) Noninvasive assessment of the digital volume pulse. Comparison with the peripheral pressure pulse. Hypertension, v. 36, p. 952.

\section{Information about the Authors}

Igor B. Isupov, Doctor of Sciences (Medicine), Professor, Department of Biology, Volgograd State University, Prosp. Universitetsky, 100, 400062 Volgograd, Russian Federation, igor.isupov.66@outlook.com.

Rimma Sh. Zatrudina, Candidate of Sciences (Physics and Mathematics), Associate Professor, Department of Laser Physics, Volgograd State University, Prosp. Universitetsky, 100, 400062 Volgograd, Russian Federation, zatrudina@yandex.ru.

\section{Информация об авторах}

Игорь Борисович Исупов, доктор медицинских наук, профессор кафедры биологии, Волгоградский государственный университет, просп. Университетский, 100, 400062 г. Волгоград, Российская Федерация, igor.isupov.66@outlook.com.

Римма Шикрулловна Затрудина, кандидат физико-математических наук, доцент кафедры лазерной физики, Волгоградский государственный университет, просп. Университетский, 100, 400062 г. Волгоград, Российская Федерация, zatrudina@yandex.ru. 